\title{
Nanonstructural Analysis of Advanced Alloys in a Local Electrode Atom Probe
}

\author{
D. W. Saxey*, J. Hanna**, R. K. Zheng*, R.K.W. Marceau*, I. Baker** and S.P. Ringer* \\ *Australian Key Centre for Microscopy \& Microanalysis, The University of Sydney, NSW, 2006, \\ Australia. \\ **The Centre for Nanomaterials Research, Thayer School of Engineering, Dartmouth College, \\ Hanover, NH 03755-8000, USA.
}

The purpose of this work is to understand the phase separation and decomposition behaviour in a new and novel range of Fe-Ni-Mn-Al spinodal alloys using the Local Electrode Atom Probe $\left(\right.$ LEAP $\left.^{\mathrm{TM}}\right)$ at the University of Sydney, where high-speed data acquisition over a large spatial and angular field of view is available. Since the installation of this instrument, we have utilised it in the analysis of spinodal decomposition, steels, $\mathrm{Al}, \mathrm{Mg}, \mathrm{Ni}$ and $\mathrm{Zr}$-based alloys. In this paper we present examples from recent work on a novel Fe-based spinodal alloy system, which exhibits a spinodal phase separation over a range of compositions and can exhibit extraordinary yield strengths of up to 2.3 GPa. Four alloys were prepared by arc melting and casting - their compositions in atomic $\%$ are listed in Fig. 1. This report is part of a broader study of the evolution of microstructure in this system, following ageing treatments at elevated temperature, where properties may be optimised. Ultimately, we intend to develop improved structure-property relationships in these types of alloys. Here it is important to note that, following the initial work of Cahn [1], most models of the functional relationship between the critical resolved shear stress (CRSS) $\sigma_{c}$, and microstructure include a strong dependence on $\lambda$, the wavelength or periodicity of the compositional fluctuations. The present results are focussed on the initial starting microstructures in the 'as-cast' condition.

Figure 1(a-d) provides histograms of the frequency distribution for the concentration of Fe over the analysed volume for each of the four alloy compositions. Figures 1(a) and (b) also include the expected binomial distribution for a random arrangement of Fe atoms. In Figs. 1(c) and (d), where the $\mathrm{Mn}: \mathrm{Al}$ ratio is higher, the profiles also include the predicted $\mathrm{Fe}$ atom distribution using the Langer/Bar-on/Miller (LBM) model for spinodal decomposition [2]. It is clear that the substitution of $\mathrm{Mn}$ for $\mathrm{Al}$ tends to enhance the spinodal decomposition.

Figure 2(a) is a typical three-dimensional (3D) atomic reconstruction of the as-cast microstructure. The wide field of view of the LEAP allows several wavelengths of the spinodal decomposition to be viewed in a single analysis. A $1 \mathrm{D}$ compositional profile from across the interface region is provided in Fig 2(b) and reveals two distinct phases separated by a narrow transition region. The large number of atoms collected also provides good statistics for quantitative analyses of the data, such as the concentration distributions. For example, the 30:20:20:30 analysis included more than 20 million ions and had a sufficient FOV in XY to show more than seven wavelengths of the decomposition. The present results reveal that the wavelength variations of the spinodal vary from a minima of $<5$ $\mathrm{nm}$ in the Fe30:Ni:20:Mn15:Al35 alloy to a maximum of between 30-60 nm in the Fe30Ni20Mn25Al25 alloy. Notwithstanding this order-of-magnitude variation in wavelength, it is significant that the hardness of the four alloys is very similar (viz.) 467 VHN. This suggests that the relationship to wavelength in these alloys is complex and further work on their mode of deformation is in progress to understand this. 


\section{References}

[1] J.W. Cahn, Acta Metall., 11 (1963) 1275.

[2] J. S. Langer, M. Bar-on and H. D. Miller, Phys. Rev. A 11, (1975) 1417.

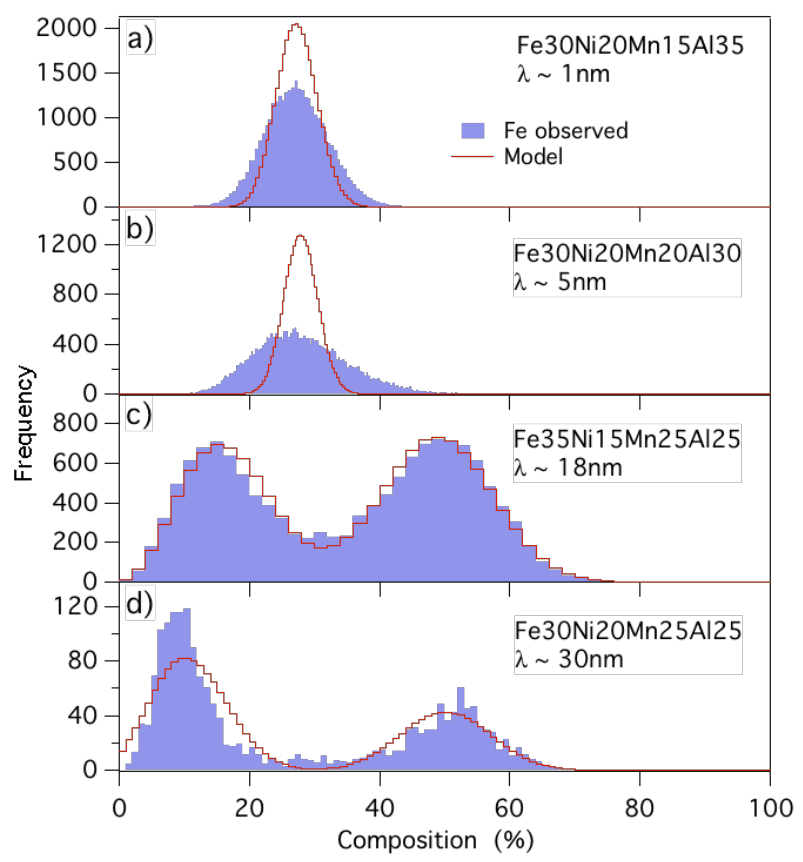

Figure 1. Frequency histograms for the concentration of Fe over the analysed volume for the four alloy compositions (shown) in the as-cast condition. The approximate wavelength of the spinodal is also provided. For (a) and (b), the binomial distribution for a random arrangement of $\mathrm{Fe}$ atoms is also provided. For (c) and (d), a fit to the data using the LBM model for spinodal decomposition [1] is provided.
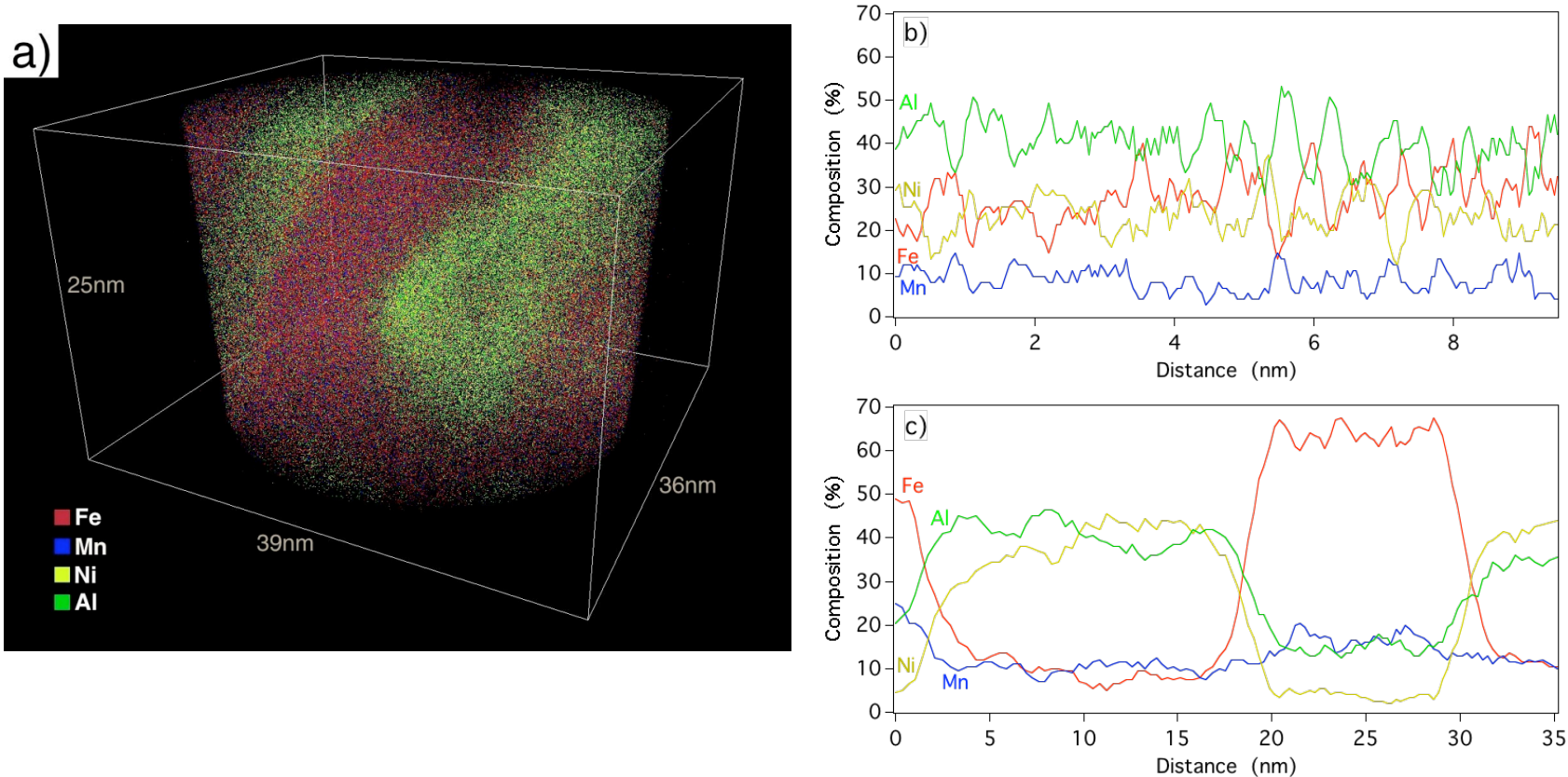

Figure 2. a) Atom map of the as-cast Fe35Ni15Mn25Al25 alloy showing the spinodal decomposition into Fe-rich and Ni-rich phases. One-dimensional composition profiles from (b) ascast Fe30:Ni:20:Mn15:Al35 and (c) as-cast Fe30Ni20Mn25Al25, providing indicative wavelength of decomposition. 